Ophthalmologe 2008 · 105:897

DOI 10.1007/s00347-008-1793-0

Online publiziert: 26. September 2008

(c) Springer Medizin Verlag 2008

\author{
S. Dithmar \\ Schwerpunkt Retinologie, Universitäts-Augenklinik Heidelberg
}

\title{
Lichtexposition bei vitreoretinaler Chirurgie
}

In den letzten Jahren konnten die Beleuchtungssysteme für die Netzhaut-/ Glaskörperchirurgie immer weiter verbessert werden, sodass die intraoperativen Ausleuchtungsmöglichkeiten heutzutage sehr gut sind. Auf dem Markt befinden sich zahlreiche Lichtquellen verschiedener Hersteller sowie eine breite Palette von Lichtleitern. Sowohl die Lichtquellen als auch die Lichtleiter unterscheiden sich voneinander zum Teil erheblich, sodass das intraoperativ ausgestrahlte Licht - je nach verwendetem System - sehr unterschiedlich sein kann.

Für den Operateur ist zunächst maßgeblich, optimale Sichtverhältnisse zu haben. Dies kann dazu führen, dass die Strahlungsenergie an der Lichtquelle entsprechend hoch eingestellt wird und es $\mathrm{zu}$ einer hohen retinalen Lichtexposition kommt. Hierdurch kann möglicherweise ein Risiko für einen Lichtschaden resultieren. Um dieses Risiko erkennen und abschätzen zu können, werden in dem Artikel von Höh et al. zunächst die physikalischen Grundlagen von Lichtstrahlung sowie die Mechanismen der Lichttoxizität wiedergegeben und auf die verschiedenen Faktoren eingegangen, die für das Ausmaß einer Lichtschädigung entscheidend sind.

In dem Artikel von Ach et al. werden verschiedene handelsübliche Lichtquellen und Lichtsonden hinsichtlich ihrer physikalischen Eigenschaften untersucht. Es zeigt sich hierbei, dass die Lichtquellen deutliche Unterschiede in den Wellenlängenspektren haben, die durch Verwendung von zuschaltbaren Filtern beeinflusst werden können. Auch die von den Lichtleitern abgestrahlte maximale Strahlungsstärke ist je nach verwendetem System sehr unterschiedlich. Dies ist von praktischer Relevanz, da die maximal tolerablen Operationszeiten in Abhängigkeit von System und Einstellungen von einigen Minuten bis hin zu mehreren Stunden schwanken können.

Herr Mittnacht erläutert in seinem Artikel die vom Gesetzgeber festgelegten Grenzwerte bzw. Richtwerte für den Einsatz von intraokularen Beleuchtungssystemen. Fast alle dieser Richtlinien wurden in jüngster Vergangenheit überarbeitet. Da die potenzielle Lichtschädigung durch intraoperative Beleuchtung von zahlreichen Faktoren abhängt, sind die vorliegenden Normen entsprechend komplex.

Das Schädigungspotenzial von intraoperativ verwendeten Lichtleitern kann auf ein Minimum reduziert werden, wenn die Lichtintensitiät reduziert wird, der Lichtleiter sich in einem möglichst großen Abstand zur Netzhaut befindet und möglichst großflächig abstrahlende Lichtsonden (Weitwinkelsonden) verwendet werden.

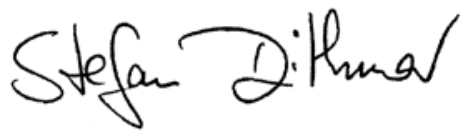

S. Dithmar

Korrespondenzadresse Prof. Dr. S. Dithmar

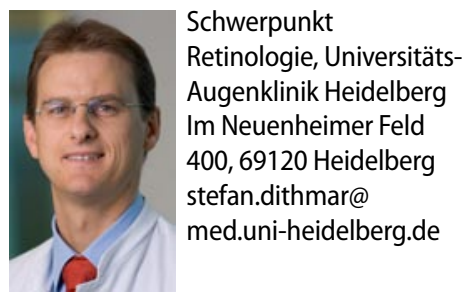

